ARCHIVO ESPAÑOL DE ARTE, LXXXVIII, 351

JULIO-SEPTIEMBRE 2015, pp. 304-309

ISSN: 0004-0428, eISSN: 1988-8511

doi: $10.3989 /$ aearte.2015.19

\title{
EL RETRATO DEL OBISPO FRANCISCO DE GAMARRA PINTADO POR ANDRÉS LÓPEZ
}

\author{
Rosalía Holgueras Arranz \\ Doctora en Historia del Arte, Universidad Complutense de Madrid
}

\begin{abstract}
El presente artículo da a conocer un retrato inédito del pintor Andrés López, uno de los seguidores de Juan Pantoja de la Cruz. Es una obra realizada a principios del siglo XVII, en la que aparece representado don Francisco de Gamarra, obispo de Ávila.

Palabras clave: Andrés López; Retrato; Francisco de Gamarra; Felipe III.
\end{abstract}

\section{THE PORTRAIT OF BISHOP FRANCISCO DE GAMARRA BY ANDRÉS LÓPEZ}

This article presents an unpublished early $17^{\text {th }}$ century portrait of Francisco de Gamarra, Bishop of Avila, painted by Andrés López, a disciple of Juan Pantoja de la Cruz.

Key words: Andrés López; Portrait; Francisco de Gamarra; Philip III.

Dedicamos este artículo a un cuadro inédito firmado por Andrés López, un retrato del obispo de Ávila don Francisco de Gamarra ${ }^{1}$, que actualmente se encuentra en el convento cisterciense de Santa Ana de la capital abulense. Este hallazgo ha permitido aumentar la larga lista de retratos conocidos que salieron de la mano de este pintor, destacado retratista de la Corte, de la nobleza y del alto clero en el primer tercio del siglo XVII.

Francisco de Gamarra nació en marzo de $1558^{2}$ en Gamarra Mayor (Álava) y, a pesar de ser un hijodalgo pobre, estudió en la Universidad de Salamanca ${ }^{3}$ bajo el patrocinio del Colegio de los Comendadores del Hábito de Alcántara, donde figura matriculado en calidad de familiar o criado. Fue nombrado capellán de su majestad Felipe III en 1599 y dos años más tarde cura de Palacio, estando durante 16 años en la Corte, donde mantuvo una vida muy activa. Formó parte de diferentes ternas para cubrir algunas diócesis vacantes, como la de Ciudad Rodrigo ${ }^{4}$, Osma ${ }^{5}$, Zamora ${ }^{6}$ y As-

\footnotetext{
${ }^{1}$ Tesis inédita de Rosalía Holgueras Arranz, título: Las Fundaciones artísticas de don Francisco de Gamarra, obispo de Ávila. Un modélico conjunto Barroco en Gamarra Mayor Álava. Universidad Complutense de Madrid, marzo de 2013.

2 AHDA-GEAH. Sig. 01124/001-01-Registros Sacramentales, Bautiz. 1549-1728. fol. 4r.

3 Libro de matrículas Archivo Histórico Universidad Salamanca (AUSA), curso 1578-79, Sig. AUSA 297, fol. 14v; curso 1579-80. Sig. AUSA 298, fol. 14; curso 1580-81 no existe; curso 1581-82. Sig. AUSA 299, fol. 11; curso 158283. Sig. AUSA 300, fol. 14; curso 1583-84. Sig. AUSA 301, fol. 16v; curso 1584-85. Sig. AUSA 302, fol. 15.

4 A.H.N. Consejos. Leg. 15211-I, 17 (1).

5 A.H.N. Consejos. Leg. 15214-I, 11 (2).

${ }^{6}$ A.H.N. Consejos. Leg. 15214-I, 13.
} 
torga $^{7}$, hasta que en 1615 se le adjudicó la de Cartagena $^{8}$ y en 1616 fue trasladado a la diócesis abulense ${ }^{9}$, donde permaneció hasta su fallecimiento en 1626.

\section{Sobre el autor}

A finales del siglo XVI, como consecuencia de la decoración del Monasterio de San Lorenzo de El Escorial, se formó una escuela pictórica en torno a Bartolomé Carducho. En este círculo cortesano, en mayor o menor medida, se cruzaron destacadas figuras en el arte de la pintura, ya fueran italianas, flamencas o españolas, entre las que encontramos a Vicente Carducho, hermano de Bartolomé, Eugenio Cajés, Juan de Roelas o Juan Pantoja de la Cruz, seguidor de Sánchez Coello, quien se convirtió a la muerte de este último en pintor de cámara de Felipe III.

Es conocido el prestigio de los pintores mencionados, pero también hay que destacar el nombre del vallisoletano Andrés López, de la escuela madrileña y uno de los seguidores de Pantoja de la Cruz. Este pintor estaba respaldado por su maestro y por don Juan Bautista de Acevedo, obispo de Valladolid, a quien realizó un retrato que se encuentra en la catedral vallisoletana. Sin embargo, en 1608 fallecieron Pantoja de la Cruz y el obispo Acevedo, lo que le dejó sin protectores en sus aspiraciones de ser nombrado pintor de Cámara a la muerte de su maestro.

Andrés López o Andrés López Polanco, como aparece citado en algunos documentos, es un pintor poco conocido dada la escasa investigación que sobre su persona y su obra se ha realizado. Fue un gran retratista, así lo demuestran algunos de los importantes encargos que le realizaron el clero y la nobleza española de la época, como el concierto que en 1618 hizo con el Conde de Lodosa para la realización de una serie de veintiocho retratos de los ascendientes de don Juan Hurtado de Mendoza Navarra y Arellano, conde de Castelnovo y Lodosa ${ }^{10}$, donde se puede leer que, “... todos los dhos veintiocho retratos an de ser cuerpos enteros en lienzos de dos baras y media..." ".1.

Más tarde recibirá otro gran encargo de don Antonio de la Cerda y Martel, quien en 1634 concertará con Andrés López catorce retratos de la Casa Austria ${ }^{12}$. Igualmente será requerida su presencia en múltiples ocasiones para hacer tasaciones ${ }^{13}$ de inventarios o de obras a término de contrato, destacando la tasación de bienes que hizo a la muerte de su maestro, Juan Pantoja de la Cruz, a petición de los herederos. Esta actividad demuestra el prestigio que tenía y su calidad como pintor y retratista.

La autora que más ha profundizado en la vida y la obra de este pintor ha sido María Kusche ${ }^{14}$, quien en su investigación recoge, describe y valora todas y cada una de las obras conocidas hasta el momento de Andrés López, a las que hay que añadir el retrato inédito del obispo abulense, Francisco de Gamarra. Entre todas las obras pone un énfasis especial en el retrato que realizó de los Infantes Carlos y Fernando (1610), del que describe los pequeños gestos que impregnan de vitalidad y ternura al cuadro, considerándolo uno de los retratos infantiles más logrados de la pintura española.

\footnotetext{
7 A.H.N. Consejos. Leg. 15214-I, 12.

${ }^{8}$ Bula para la Diócesis de Cartagena. http://aer.mcu.es/sgae/index_aer.jsp - ES.47161.AGS/2.30//Patronato Real, caja 67 , doc. 63.

${ }^{9}$ Bula para la Diócesis de Cartagena. http://aer.mcu.es/sgae/index_aer.jsp - ES.47161.AGS/2.30//Patronato Real, caja 65 , doc. 101.

${ }^{10}$ Kusche Zettelmeyer, 2007: p. 420.

11 A.H.P.M. Esc. García, Francisco. Prot. 2407, año 1618, fol. 5.

12 A.H.P.M. Esc. Cerón, Diego. Prot. 4168, año 1634, fol. 137.

13 A.H.P.M. Esc. Obregón, Diego de. Prot. 3795, año 1629, fols. 106-108. Esc. Medina, Francisco de. Prot. 7366, fol. 501. Esc. Portero, Alonso. Prot. 6446, fols. 48-49.

${ }^{14}$ Kusche Zettelmeyer, 2007: p. 407-449, 576,577.
}

Arch. esp. arte, LXXXVIII, 351, JULIO-SEPTIEMBRE 2015, 304-309

ISSN: 0004-0428, eISSN: 1988-8511, doi: 10.3989/aearte.2015.19 
Este pintor también realizó retratos de Felipe III y de su esposa Margarita de Austria, aunque se trataría de copias de otros pintores como Juan Pantoja de la Cruz o Bartolomé González, pues Andrés López no tuvo acceso al posado natural de los monarcas. En estas obras lo que hizo fueron representaciones reinventadas, ya que no se limitaba a copiar, sino que interpretaba los modelos a su manera dándoles una visión muy personal.

\section{Sobre el retrato}

Se trata de una pintura al óleo sobre lienzo, de dos varas y media de alto por una vara y poco más de tercia de ancho $(207 \times 116 \mathrm{~cm})$, que conserva el marco de la época de color negro decorado con motivos vegetales dorados en las esquinas y en el centro de cada lado. Es una representación de cuerpo entero que sigue el esquema del retrato cortesano (fig. 1).

Don Francisco de Gamarra viste indumentaria de presbítero, bonete, sotana y muceta de doctor,

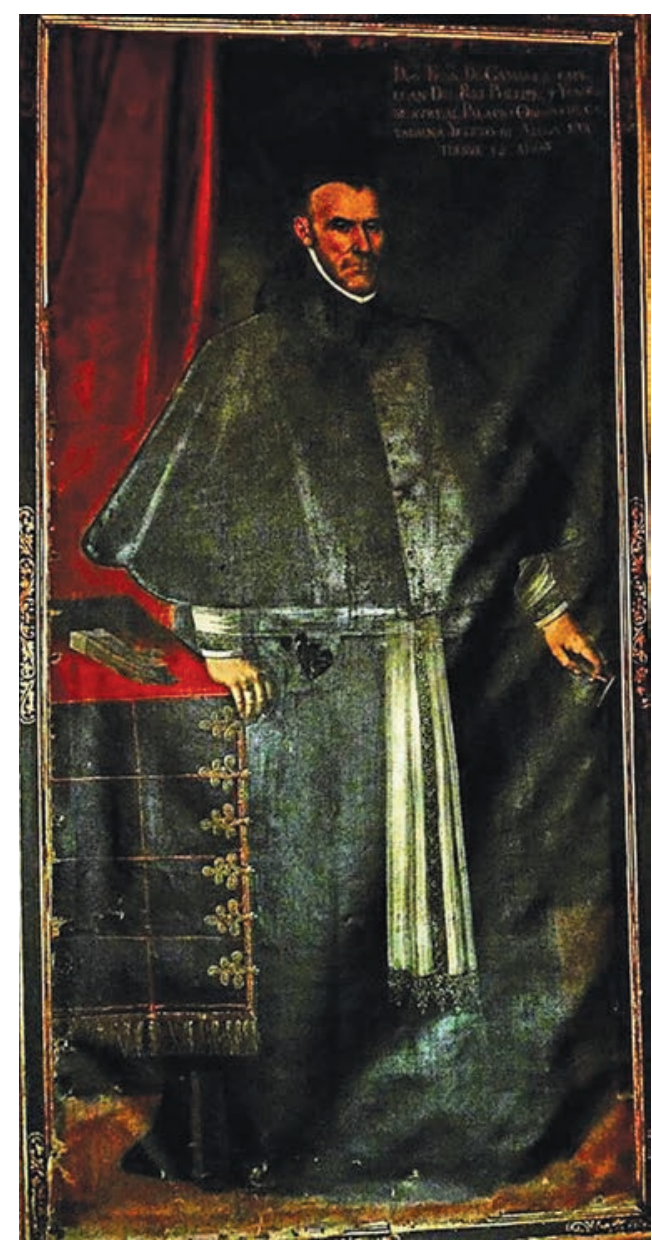

Fig. 1. Francisco de Gamarra, óleo sobre lienzo obra de Andrés López c. 1616? Convento Cisterciense de Santa Ana (Ávila). todo de color negro, con roquete y cinturón de seda blanco; está representado de pie, en una postura que muestra cierto distanciamiento, elegancia y dignidad, como si quisiera destacar y hacer ver al espectador el rango al que pertenece. Hay un bufete cubierto por una tapicería lisa de terciopelo de color rojo en la parte superior, mientras que el frontal es negro y aparece dividido en cuadrados y rematado con flecos dorados, al igual que los adornos y el clavazón. Sobre el bufete se encuentran unos guantes de piel, un elemento que denota distinción, poder y elegancia.

Posa la mano derecha en el bufete mostrando el anillo de oro que lleva en el dedo, el anillo de doctor, mientras que con la mano izquierda sostiene un libro entreabierto queriendo decir con ello que es un hombre culto y que estaba leyendo (fig. 2). A la izquierda del cuadro, sobre el bufete, cuelga una discreta cortina de terciopelo rojo con pliegues tubulares y bajo él, en una zona oscurecida, encontramos la firma del autor "andres lopez f" (fig. 3).

En esta pintura, Andrés López, consigue por medio de la degradación del negro, una escala cromática que sugiere la textura y la calidad de los tejidos, creando un efecto óptico que permite imaginar la suavidad de la seda y distinguir la botonadura forrada de la muceta. Los puntos de luz a través del color blanco se concentran en la fina línea que asoma en el cuello, en las mangas y en las caídas del cinturón de seda; en ellas, al igual que en los puños de las mangas, podemos apreciar una fina pasamanería bordada en blanco sobre el fondo negro, y un minucioso encaje rematando las caídas del cinturón, también en blanco. 


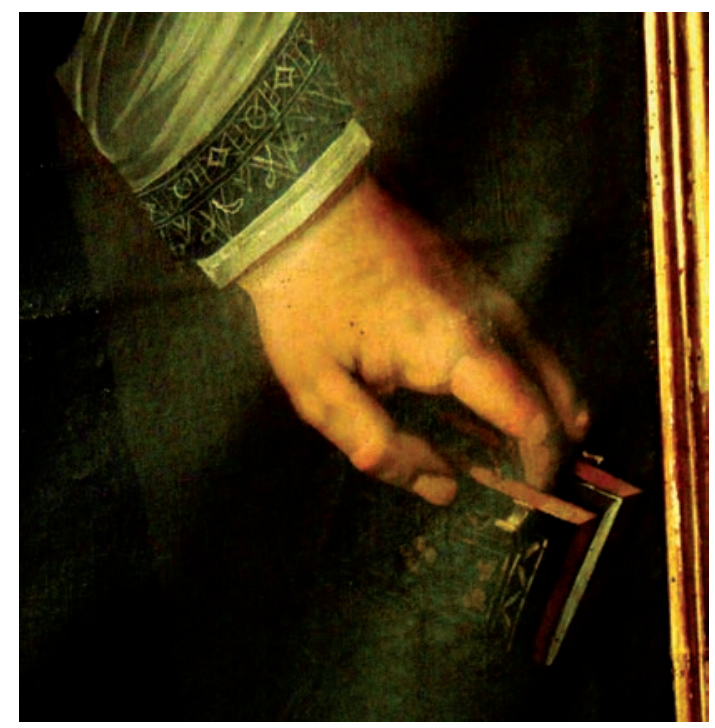

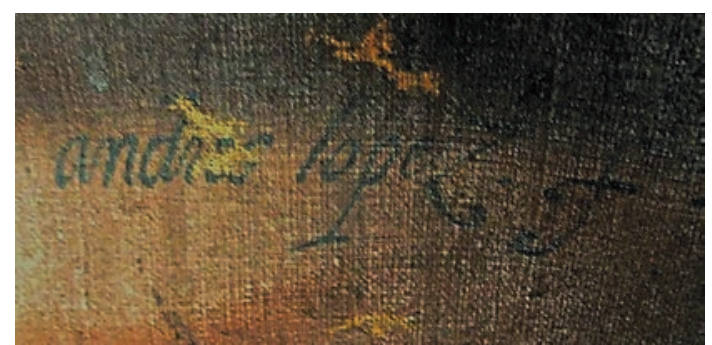

Fig. 2. Detalle, firma del autor "andres lopez f".

Fig. 3. Detalle, del libro entreabierto.

Es un retrato en el que Andrés López utiliza elementos como el bufete o la cortina para resaltar y reforzar la distinción del personaje, mostrando al observador el rango social al que pertenece. Son recursos muy habituales en los retratos realizados por los pintores que se movían en los círculos cercanos a la Corte a finales del siglo XVI y principios del XVII. Es el caso de Sánchez Coello en su retrato del Infante don Carlos, de Pantoja de la Cruz en el del Infante don Felipe con armadura, o de Bartolomé González en el retrato del Infante don Fernando de Austria, entre otros muchos. El propio Andrés López utiliza esos complementos en retratos posteriores, como el que hace de Felipe III en negro (1617), y en el del joven santiaguista (1619-1620).

El fondo del cuadro es de color neutro más oscuro que el suelo liso de color terroso, destacando en la parte superior derecha una inscripción en la que se puede leer:

\author{
DON FRAN ${ }^{\text {co }}$ DE GAMARRA CAPE \\ LLAN DEL REI PHELIPE 3 Y CURA \\ DE SU REAL PALACIO OBISPO DE CAR \\ TAGENA Y ELETO DE ABILA ETA \\ TIS SUE 58 AÑOS
}

Si nos atenemos a lo que pone en la misma, dice que el personaje tiene 58 años, es obispo de Cartagena y electo de Ávila, como efectivamente así era en el primer tercio del siglo XVII, tenemos que datar la obra en el segundo cuarto de 1616, más concretamente entre el 23 de marzo, fecha de la onomástica de don Francisco de Gamarra, y finales del mes de mayo, momento en el que se confirmó su nombramiento como obispo de Ávila. Sin embargo, sorprende que aparezca vestido de presbítero cuando era obispo desde 1615, y más teniendo en cuenta que cuando un personaje se hacía un retrato de estas características, su objetivo era mostrar a los demás el estatus social alcanzado debido a la importancia que se daba a las apariencias.

Todo induce a pensar que el retrato estuviera realizado con anterioridad a 1615, cuando aún era cura de palacio y se movía por la Corte coincidiendo con Andrés López, pintor, y que sería con posterioridad cuando don Francisco de Gamarra mandara incluir la inscripción para dejar constancia del nuevo rango de obispo.

Arch. esp. arte, LXXXVIII, 351, JULIO-SEPTIEMBRE 2015, 304-309

ISSN: 0004-0428, eISSN: 1988-8511, doi: 10.3989/aearte.2015.19 
El cuadro se encuentra muy deteriorado con pérdidas de pigmentos y roturas en el bastidor. Una adecuada acción restauradora del mismo permitiría su mejor conservación y poder constatar si la inscripción está añadida con posterioridad o si pertenece al momento de la realización del retrato.

\section{BIBLIOGRAFÍA}

Agulló y Cobo, Mercedes (1994): Documentos para la Historia de la Pintura Española I. Museo del Prado. Madrid.

Agulló y Cobo, Mercedes (1981): Más noticias sobre pintores madrileños de los siglos XVI al XVIII. Madrid. Ayuntamiento de Madrid Delegación de Cultura.

Agulló y Cobo, Mercedes (1978): Noticias sobre pintores madrileños de los siglos XVI y XVII. Granada: Universidad de Granada.

Caturla, María Luisa (1956): “Andrés López Polanco”. En: Cuadernos de Estudios Gallegos. Vol. XI, Fasc. XXXV, Santiago de Compostela. pp. 389-405.

Kusche Zettelmeyer, María (2004): "El caballero cristiano y su dama: El retrato de representación de cuerpo entero". En: Cuadernos de Arte e Iconografía. Tomo 13, No 25, pp. 3-102. Título original: Der Christliche Ritter und seine Dame, das Repräsentationsbidnis in ganzer Figur. Munich 1991. Traducido por la autora.

Kusche Zettelmeyer, María (2007): Juan Pantoja de la Cruz y sus seguidores: B. González, R. de Villandrando y A. López Polanco. Madrid. Ed. Fundación de Arte Hispánico.

Lavín Berdonces, Ana Carmen (2012): "Un retrato desconocido de Alonso Sánchez Coello: El obispo D. Diego de Cobarrubias" (Museo del Castillo de Perelada, Gerona). En: Archivo Español de Arte, LXXXVI, 341, enero-marzo, pp. 49-76.

Martínez Millán, José / Visceglia, María Antonieta (dirs.) (2008): La Monarquia de Felipe III: La casa del Rey. Vol. I, II, III. Madrid. Fundación Mafre, Instituto de Cultura.

Miquel Juan, Matilde (2012): "Un retrato inédito del Cardenal Alfonso de Borja en una tabla mariana de Gonçal Peris Sarriá". En: Archivo Español de Arte, LXXXV, 340. octubre-diciembre, pp. 351-387.

Morán Turina, Miguel (1997): "Los gustos pictóricos en la corte de Felipe III". En: El arte de mirar. La pintura y su público en la España de Velázquez. Madrid. Ed. Istmo.

Muñoz y Manzano, Cipriano (conde de la Viñaza) (1889): Adiciones al diccionario histórico de los más ilustres profesores de las bellas artes en España, de don Juan Agustín Ceán Bermúdez. T.I., Madrid.

Negredo del Cerro, Fernando (2006): "La capilla de palacio a principios del siglo XVII Otras formas de poder en el Alcázar madrileño". En: Studia Histórica. Historia Moderna. Vol. 28. Salamanca: Ed. Universidad de Salamanca.

Pérez Sánchez, Alfonso Emilio (1968): "La crisis de la pintura en torno a 1600”. En: España en las crisis del arte europeo. Coloquios celebrados en conmemoración de los XXV años de la fundación del Consejo Superior de Investigaciones Científicas. Madrid. Ed. Casa de Velázquez, pp. 167-178.

Pérez Sánchez, Alfonso Emilio (2010): Pintura barroca en España. (1600-1750). Madrid. Ed. Actualizada por Benito Navarrete Prieto. Manuales Arte Cátedra.

Portela Sandoval, Francisco José (1997): "La Escultura y la Pintura en el Museo del Ejército". En Militaria. Revista de cultura militar. No 9. Madrid: Servicio de Publicaciones Universidad Complutense de Madrid, pp. 121-138.

Portús Pérez, Javier (2007): "El siglo XVII: la madurez del género". En: Ruiz Gómez, Leticia (com.). El retrato español en el Prado: Del Greco a Goya. Madrid. Ed. Museo Nacional del Prado. pp. 83-89.

Ruiz Gómez, Leticia (2005): "Retratos de corte en la monarquía española (1530-1660)”. González Escribano, Raquel; Villarreal Gato, Lucía (Coord.); Portús Pérez, Javier (Comisario). El retrato español: Del Greco a Picasso. Madrid. Ed. Museo Nacional del Prado. pp. 91-119.

Ruiz Gómez, Leticia (com.) (2007): "El retrato español en el Museo del Prado (1490-1800)”. En: El retrato español en el Prado: Del Greco a Goya. Madrid. Ed. Museo Nacional del Prado. pp. 17-36.

Arch. esp. arte, LXXXVIII, 351, JULIO-SEPTIEMBRE 2015, 304-309 ISSN: 0004-0428, eISSN: 1988-8511, doi: 10.3989/aearte.2015.19 
Ruiz Gómez, Leticia (com.) (2007): “La creación del retrato español en el siglo XVI". En: El retrato español en el Prado: Del Greco a Goya. Madrid. Ed. Museo Nacional del Prado. pp. 37-43.

Urrea Fernández, Jesús (1985): “Enrique Trozo y Andrés López, retratistas de obispos”. En: Boletín del Seminario de Estudios de Arte y Arqueología: BSAA. T. 51. pp. 483-487.

Urrea Fernández, Jesús (2001): "El Conde Duque de Olivares". En: Urrea Fernández, Jesús (Director) Pintura del Museo Nacional de Escultura siglos XV al XVIII. Santander: Caja Cantabria, pp. 69-71.

Valdivieso González, Enrique (1999): "Barroco y Rococó: La Pintura". En: Ramírez Domínguez, Juan Antonio (Director) Historia del Arte: La Edad Moderna. Madrid. Ed. Alianza Editorial S.A. pp. 252-263.

Fecha de recepción: 20-V-2014

Fecha de aceptación: 10-X-2014

Arch. esp. arte, LXXXVIII, 351, JULIO-SEPTIEMBRE 2015, 304-309

ISSN: 0004-0428, eISSN: 1988-8511, doi: 10.3989/aearte.2015.19 